\title{
The Models Of The Redundant Transmission Through The Aggregated Channels
}

\author{
Vladimir Bogatyrev \\ Department of Computation Technologies \\ ITMO University \\ St. Petersburg, Russia \\ vladimir.bogatyrev@gmail.com
}

\author{
Ivan Slastikhin \\ Department of Computation Technologies \\ ITMO University \\ St. Petersburg, Russia \\ stopgo89@gmail.com
}

\begin{abstract}
The paper deals with the distributed systems with channel aggregation. The aim of the work is to investigate the possibilities of increasing the efficiency of the exchange through the aggregated channels while making the redundant transmissions of the packet copies that are critical to the timing of error-free delivery to an addressee.
\end{abstract}

The study of the exchange effectiveness is based on the simulation modeling in the AnyLogic 7 environment.

We consider the variants of the exchange arrangement when the set of the channels is divided into groups with the implementation of the single multiple access procedure for each group or without division into groups with implementation of the single multiple access procedure separately for each channel. The efficiency of the redundant transmissions of the packet copies through the aggregated channels is shown for the different variants of the queue organization, multiple access and channel grouping. The limits of the redundant transmission expediency of packets that are critical to the timing of the error-free delivery through the aggregated channels are determined.

Keywords - aggregated channels, multiple access, redundant transmission, simulation modeling

\section{INTRODUCTION}

High reliability, fault tolerance, performance and security [1-5] of the distributed computing systems and networks [6] of various purposes [7-9] are achieved by reserving and consolidating the resources of computing nodes and communication channels [10-18].

Studies of the resource consolidation effectiveness in the transmission of data through the aggregated channels in the organization of common queues to each channel separately, to all channels simultaneously or to the channels divided into groups were carried out in articles [19-21].

In the article [22] it is proposed to organize an exchange when channels are combined into groups with the implementation of the general request queue for each group and a single multiple access procedure for all channels of the group. Resources of all channels of one group are simultaneously provided as a result of the multiple access procedure for each request. When a node is granted an access to a channel group, the packet from its general queue is copied and each reserved copy is transmitted through a separate channel of the group.

The redundant transmission of packets makes it possible to increase the probability of error-free delivery of at least one copy of the packet to the addressee and to reduce the retransmissions costs of erroneously delivered packets. At the same time, the transmission of packet copies increases the load of channels and can lead to an increase in the time of the packet stay in the system and, as a result, to a possible decrease in the delivery probability of packets for the maximum allowable time. This technical contradiction makes it necessary to conduct additional studies, including those ones aimed at determining the area (boundary) of the efficient reservation of the packet copies via the aggregated channels.

The researches of the redundant transmission efficiency were carried out in the articles [12-22] on the basis of the analytical modeling limited to the simplest queuing models and did not allow to take into account the influence of multiple access on the redundant transmission efficiency.

The aim of the work is to develop the simulation models focused on the research of possibilities for increasing the exchange efficiency through the aggregated channels while copy transmission reserving of time-critical error-free package delivery to the addressee, taking into account the division of the channel resources into groups with different versions of the multiple access organization.

With reserving the transmissions through the aggregated channels let's consider the options:

- with the division of channels into groups, the number of channels in each group corresponds to the multiplicity of the redundant transmission, all the channels, which are included into a certain group are simultaneously allocated for simultaneous transmission of all packets copies as a result of implementing a multiple access procedure for this group.

- without uniting the channels into groups, when the multiple access procedure is implemented for each channel separately, as a result of which the channels, which are allocated for transmitting the copies of requests, are not provided simultaneously and the transmission of the packets copies will also not be simultaneous.

The developed simulation models for supporting the choice of the designed solutions for the organization of exchange through the redundant channels are implemented in the AnyLogic 7 environment. 


\section{Building The Imitation Model of Data TRANSMISSION WITH CHANNELS CONSOLIDATION}

Let's present the simulation models of systems with channel aggregation for various multiples of redundant transmissions in the case of combining channels into the groups with the implementation of a single multiple access procedure for each allocated channel group.

As a basic, let us consider the option of the exchange organization without the redundant data transmission.

In order to determine the efficiency of the redundant transmissions let's consider the systems:

- with duplicated transmissions when channels are divided into groups of 2 channels each;

- with splitting channels into groups of 4 channels each with the transmission of four packet copies;

- with the combination of all channels in one group with the transmission on each channel of a separate packet copy.

We assume that a single multiple access procedure is implemented for each group.

Fig. 1 shows a simulation model of the data transmission system without the redundant transmission. The source block is the source of the packets. The queue block is an infinite single queue of packets. The delay1, delay $2, \ldots$, delay 8 blocks simulate 8 identical communication channels. The hold block reflects the blocking of packet transmission, when all channels are busy. The selectOutput 1 and selectOutput2 blocks reflect the distribution of packets between the free communication channels. The DelayWi blocks simulate the delays in providing the channel with the multiple access for the $i$-th group of the aggregated channels. The checkErrori blocks model the validation of the data transmission for the $i$-th group of the aggregated channels. The sinki and sinkFi blocks are used to count the number of packets through the $i$-th channel with an error and without an error respectively.

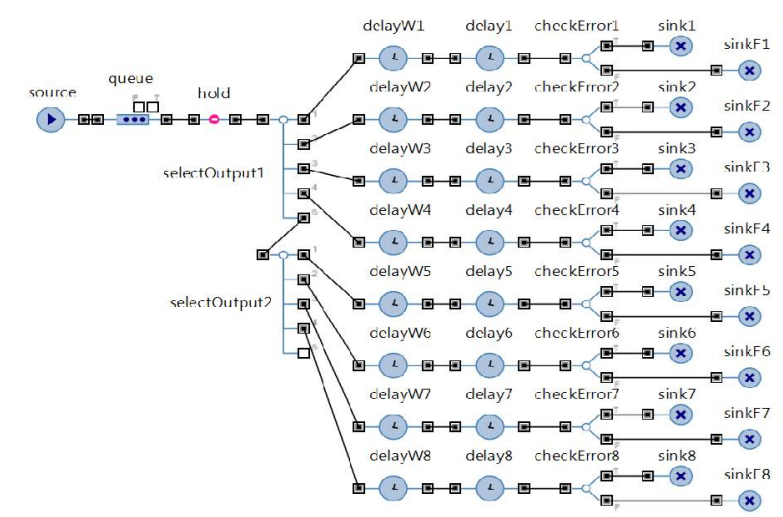

Fig. 1. The simulation model of the data transmission system without the redundant transmissions.

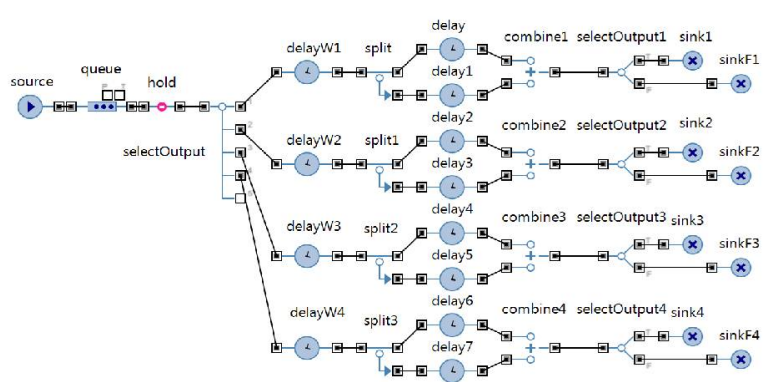

Fig. 2. The simulation model of the data transmission system with the transmission redundancy of multiplicity 2 .

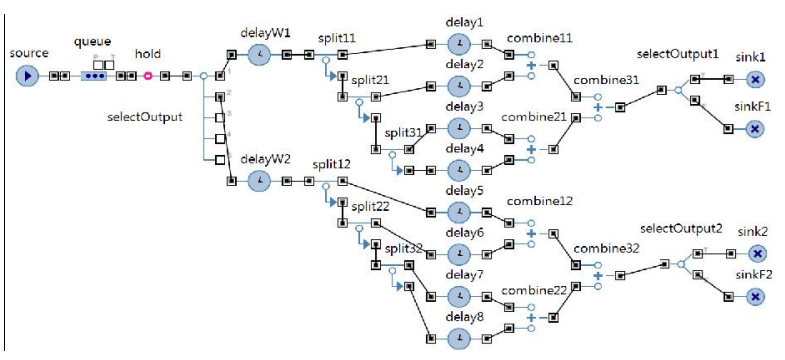

Fig. 3. The simulation model of the data transmission system with the transmission redundancy of multiplicity 4 .

Fig. 2 shows the model of the data transmission system with communication channel clustering into groups of 2 channels. In this model, the blocks split, split1, split2, split3 are added. They reflect the copying of the packet received to the transmission. The time is not spent on copying the packet. The combine, combine1, combine2, combine3 blocks reflect the validation of the delivery of at least one of the packet copies. Delays in the provision of channel group resources as a result of the multiple access are simulated by means of delay $W i$ blocks for the $i$-th group of the aggregated channels.

Fig. 3 shows the model of the data transmission system with communication channel clustering into groups of 4 channels. In this model the blocks split $1 i$, split $2 i$, split $3 i$ are additionally introduced. They reflect the copying of the incoming packet to the $i$-th group of the aggregated channels. The combine $i$, combine $2 i$, combine $3 i$ blocks reflect the validation of the delivery of at least one copy of the packet in the $i$-th group of the aggregated channels.

\section{The Results of the Simulation Studies ON THE \\ EFFECTIVENESS EVALUATION OF THE REDUNDANT Transmission Through the Aggregated Channels}

The redundant transmission is considered to be successful if at least one copy of the package is delivered faultless. 


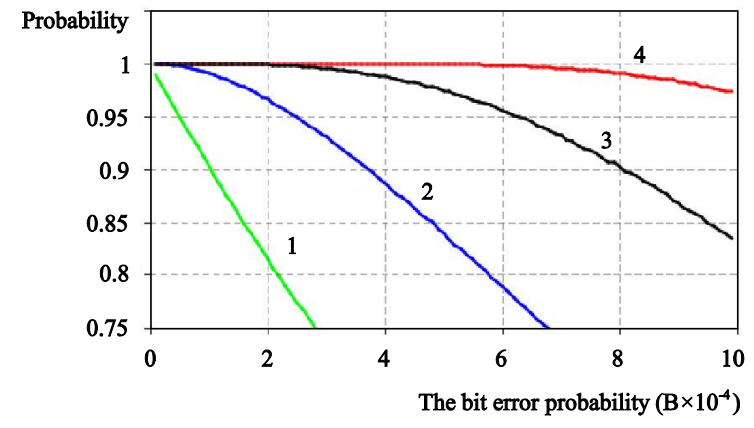

Fig. 4. The probability of the error-free transmission from the probability of bit errors.

Fig. 4 shows the result obtained during the simulation experiments, the probability of the error-free packet delivery to a recipient of the bit error rate. The simulation experiments were performed with the packet arrival rate of $\lambda$ $=300001 / \mathrm{s}$, the channel transmission rate of $L=8388608$ bits per second, the length of the incoming packets of $N=$ 1024 bits and the variation of the bit error probability $B$ from $1 \times 10^{-5}$ to $1 \times 10^{-3}$ in steps of $1 \times 10^{-5}$.

Curves 1, 2, 3, 4 reflect the probabilities of the error-free delivery in the data transmission organization with the multiples of $\mathrm{k}=1,2,4,8$ respectively. As it can be seen from the presented chart the redundant packet transmission contributes to the increase of the error-free delivery probability.

In addition to the increasing probability of the error-free delivery of a copy packet at least through one of the channels the reservation of packet copies, the increase in the reservation multiplicity of the transmitted packet copies leads to the increase in the load of channels and the increase in the average waiting time and may lead to their nondelivery to the addressee for the maximum allowable time. It can lead to the destruction of packets, which exceeded the permissible "lifetime".

The efficiency of the system's operation, taking into account the delay and the probability of delivery, is determined by the criterion expressing the average margin for the elapsed time of the error-free packet delivery calculated as

$$
M=P\left(t_{0}-T\right)
$$

Where $P$ is the packet delivery probability, which can be defined as $P=N_{l} / N_{0}$, where $N_{l}$ is the number of packets correctly delivered to the destination, and $N_{0}$ is the number of packets sent; $T_{0}$ is the time of the allowed delay in the packet transmission; $T$ is the average time of the package stay in the system.

The experiments were carried out to evaluate the efficiency of the redundant transmissions for various reservation

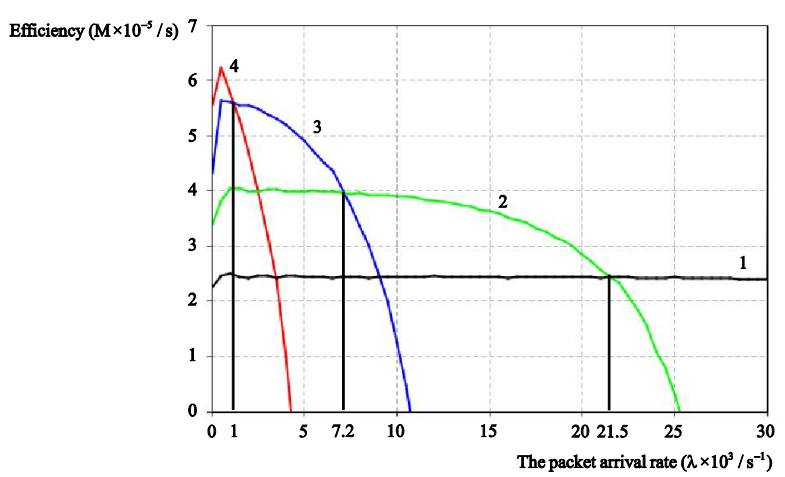

Fig. 5. Dependence of the efficiency on the intensity of applications $\lambda$.

multiplicities $k=1,2,4,8$. While carrying out the simulation experiments the transmission rate of the channels is given by $L=8388608$ bits per second, the length of the incoming packets $N=1024$ bits, the time of the admissible delay $t_{0}=2 \times 10^{-4} \mathrm{~s}$, the bit error probability $B=1 \times 10^{-3}$.

Fig 5 shows the dependence of the considered efficiency criterion $M$ on the intensity of the packet arrival $\lambda$. Curves 1 , $2,3,4$ correspond to the multiplicity of the redundant transmissions $k=1,2,4,8$.

As it can be seen from the presented chart there is the existence of the expedient application field of the redundant transmission for different backup multiplicities. The redundant transmission with the multiplicity of 8 is suitable for the low-intensity applications. The transmission becomes more efficient with the reduction of the reservation multiplicity, with the increase in the intensity of the packet flow. When the intensity of the request flow is high the use of reservations becomes useless.

\section{The Analytical Model of the Data Transmission THROUGH THE AGGREGATED CHANNELS WHILE THEIR COMBINING INTO GROUPS}

During the packet transmission through the communication channels, individual node queues are combined into a common queue for multiple access implementing. When the channels are reserved, the general queue is organized to the whole set of channels, each of which is treated as a service device. At the same time, if the delays in the process of organizing multiple access are neglected, then the system is represented as a multi-channel queuing system (QMS) with a common queue.

Consider the case of dividing a set of channels into the groups of $\mathrm{k}$ channels for the redundant transmission of packets copies. In view of the channels identity and simultaneous transmission over the $k$ channels of one group of the $k$ backup copies of packets, let's represent each group of channels as a single serving device. The total number of 
serving devices in the QMS will thus be $m=G / k$ (we will

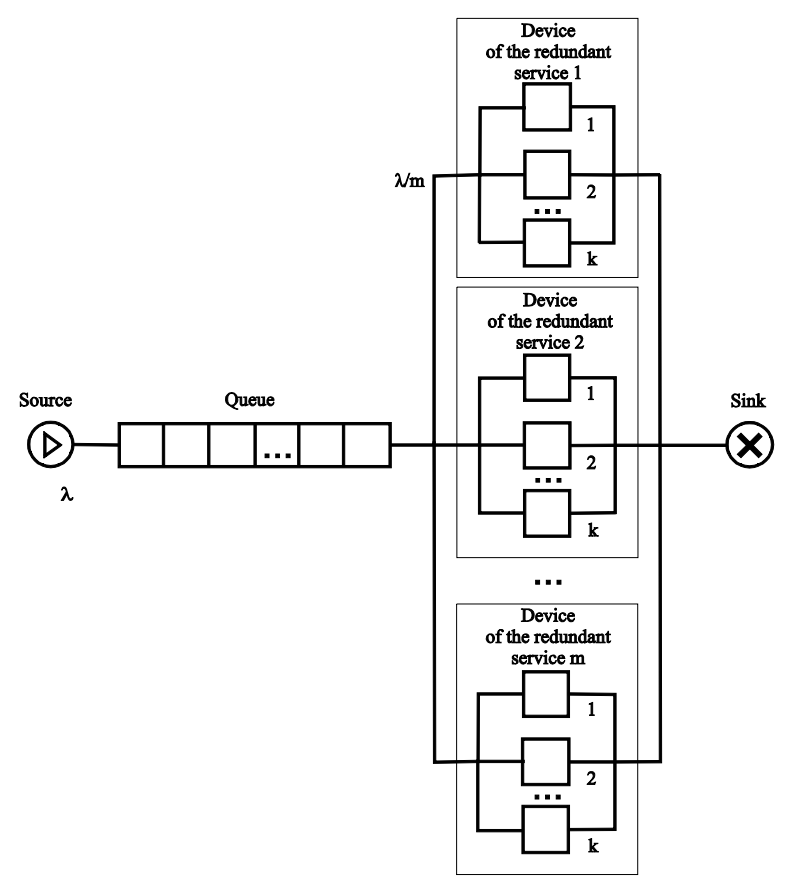

Fig. 6. The service model for linking channels into the groups for the redundant transmission of packets copies.

assume that $G / k$ is an integer) [22].

The service model for linking channels into the groups for the redundant transmission of packets copies is shown in Fig. 6.

The proposed system representation (Fig. 6) allows to determine the average time of each packet copy stay in the system by the formula for the multichannel QMS of the type $\mathrm{M} / \mathrm{M} / \mathrm{m}$ as [6]:

$$
T=P_{0} v / m(1-\rho)
$$

Where $\rho=\lambda v / m$ is the system load factor, $v=N / L$ is the average packet transmission time; $N$ is the packet length in bits; $L$ is the speed of the data channel, $\lambda$ is the intensity of the requests, $P_{0}$ is the probability that the request finds an empty queue

$$
P_{0}=\frac{(m \rho)^{m}}{m !(1-\rho)}\left(\sum_{s=0}^{m-1} \frac{(m \rho)^{m}}{s !}+\frac{(m \rho)^{m}}{m !(1-\rho)}\right)^{-1}
$$

The efficiency of the packet transmission through the aggregated channels, as well as in simulation modeling, is defined as $M(1)$. If the transmission of the packet is simple (without reservation), then $m=G$.

When transmitting the packet without reservation, the probability of its error-free delivery is

$$
P=(1-B)^{N}
$$

where $B$ is the probability of the bit error in

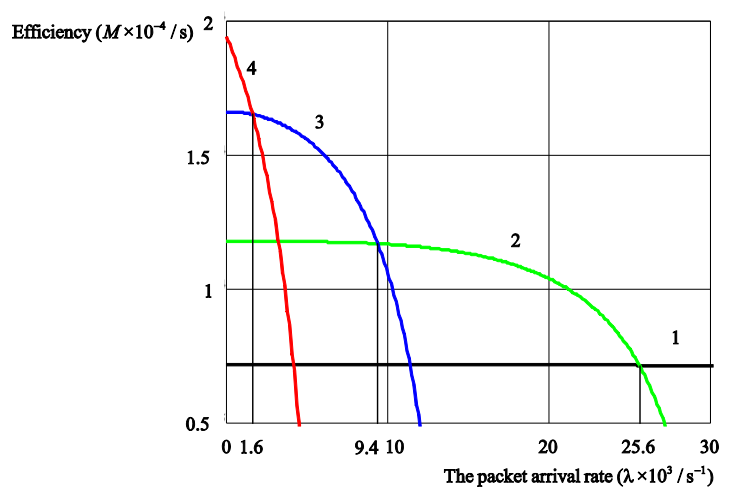

Fig. 7. Dependence of the efficiency of the redundant packet transmissions on the intensity of applications $\lambda$, obtained by the analytical modeling.

transmission.

In the case of the redundant transmission, the probability of the error-free delivery, provided that the packet transmission is considered successful if at least one of its copies is unmistakably transmitted [23] is defined as

$$
P=1-\left(1-(1-B)^{N}\right)^{k}
$$

Fig. 7 shows the dependence of the efficiency $M$ of the redundant packet transmissions on the intensity of requests for the packet transmission through $G=8$ channels. The calculation was carried out at $N=1024$ bits; $L=8388608$ $\mathrm{bit} / \mathrm{s} ; B=10^{-3} ; t_{0}=2 \times 10^{-4} \mathrm{~s}$. Curves $1,2,3,4$ correspond to the results of the analytical modeling at the transmission reserve multiplicities $k=1,2,4,8$.

The chart shows the existence of the efficiency area of the redundant packet transmissions. So, with the load of less than $16001 / \mathrm{s}$, the best results are shown in the transmission of eight copies of the packets, from $1600 \mathrm{1} / \mathrm{s}$ to $9400 \mathrm{1} / \mathrm{s}$, it is expedient to transfer four copies, from $9400 \mathrm{1} / \mathrm{s}$ to 25600 $1 / \mathrm{s}$, it is expedient to transfer two copies, and more than $256001 / \mathrm{s}$ - transmission of packets without redundancy.

The difference between the values of the criterion $M$, obtained by the analytical and simulation modeling, will be denoted by $D$. The results of the estimation of $D$ are shown in Fig. 8, in which curves 1, 2, 3, 4 correspond to the difference between the analytical and simulation modeling for $k=1,2,4,8$. Fig. 5, 7, 8 confirm the similarity of the results obtained in the simulation and analytical models. It should be noted that the results of the analytical modeling do not take into account the effects of multiple access. 


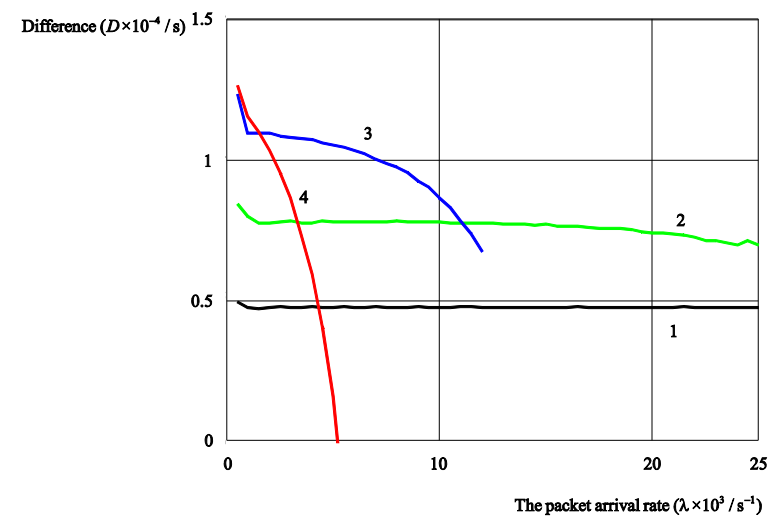

Fig. 8. The difference between the values of the criterion M, obtained in the analytical and simulation modelling.

\section{Building the Imitation Model of the Data TRANSMISSION WITHOUT COMBINING CHANNELS INTO GROUPS}

The multiple access procedure is carried out separately for each channel.

Fig. 9 shows the simulation model of the system with the realization of separate access to various channels. The Sourcei blocks are the sources of the packets. The queuei blocks are infinite queues of packets for the $i$-th source. The spliti blocks are responsible for creating the required number of copies of the packet for the $i$-th source. The delay 1 , delay $2, \ldots$, delay 8 blocks simulate 8 identical communication channels. The selectOutputIn and selectOutputOuti blocks reflect the distribution of packets between the free communication channels. The delay $W$ and hold blocks simulate the delays during the channel provides for the multiple access. The checkErrori blocks model the validation of the data transmission for the $i$-th channel. The sinki and sinkFi blocks are used to count the number of packets through the $i$-th channel with an error and with an error respectively.

When a package arrives, the required number of the package copies is generated, while the time for creating

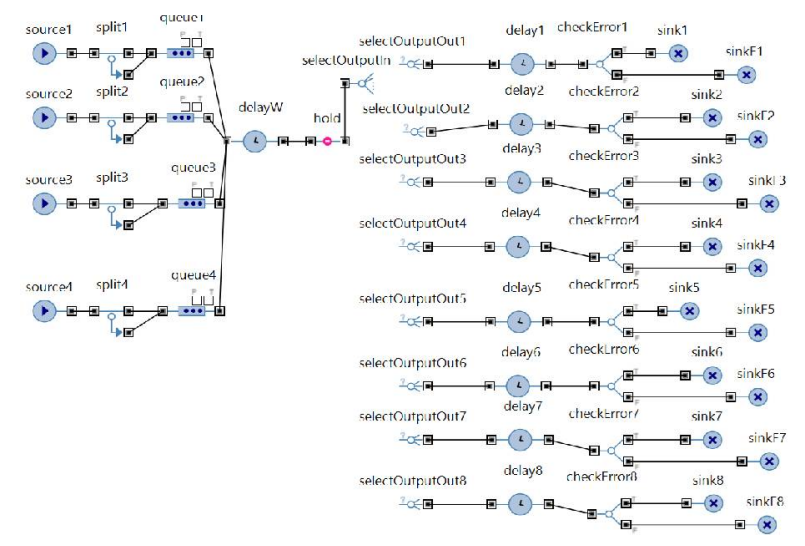

Fig. 9. The simulation model data transmission system without combining channels into groups.

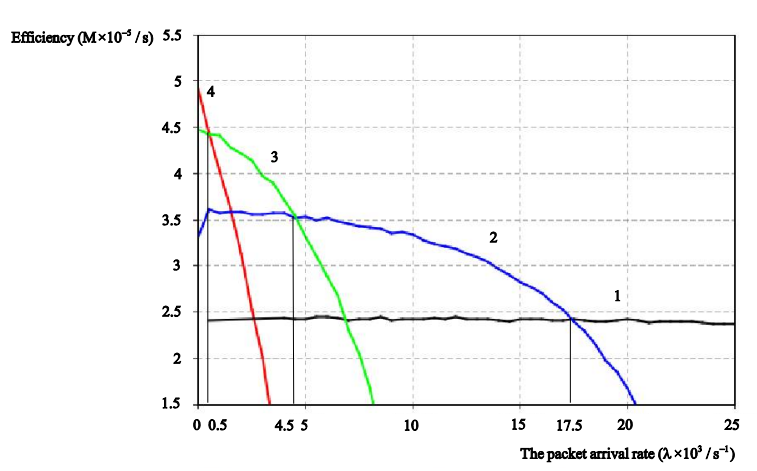

Fig. 10. The dependance of the efficiency of redundant packet transmissions on the intensity of requests without combining channels into groups.

copies is not wasted. The copies are placed in the appropriate queues. The transmission is carried out through the free channels with the organization of the multiple access to each channel separately. If at least one copy of the packet is successfully transmitted, the remaining copies of this package are removed from the queues.

The efficiency of the system functioning is defined by the criterion $M(1)$. The experiments were carried out to evaluate the efficiency of the redundant transmissions for various number of copies $n=0,1,3,7$. While carrying out the simulation experiments the transmission rate of the channels is given by $L=8388608$ bits per second, the length of the incoming packets $N=1024$ bits, the time of the admissible delay $t_{0}=2 \times 10^{-4}$ seconds, the bit error probability $B=10^{-3}$

Fig. 10 shows the dependence of the considered efficiency criterion $M$ on the intensity of the packet arrival $\lambda$. Curves 1, 2, 3, 4 correspond to the certain number of copies $n=0,1,3,7$.

As it can be seen from the presented chart there is the existence of the expedient application field of the redundant transmission for different backup multiplicities. With the increase of the packet flow intensity the transmission becomes more efficient when the reservation multiplicity is reduced. When the intensity request flow is high the use of reservations becomes useless.

\section{CONCLUSION}

Thus, the simulation models of the redundant transmissions of the packet copies through the aggregated channels are proposed with the possibility of their combining into groups with the implementation of the uniform procedure for the multiple access and without such possibility with the multiple access for each channel separately.

The effectiveness of the redundant transmission organization of the packet copies through the aggregated 
channels with their combining into groups and without their combining is shown.

The boundaries of the expedient use of the redundant packet transmission, critical to the time of their error-free delivery are determined.

\section{REFERENCES}

[1] Aysan, H., Fault-Tolerance Strategies and Probabilistic Guarantees for Real-Time Systems, Mälardalen Univ., Västerås, Sweden, 2012.

[2] Sheng-Tzong Cheng, Chia-Mei Chen., and Tripathic, S.K., Faulttolerance model for multiprocessor real-time systems, J. Compt. Syst. Sci., 2000, vol. 61, pp. 457--477.

[3] Kopetz, H., Real-Time Systems: Design Principles for Distributed Embedded Applications, Springer-Verlag, 2011.

[4] Shooman, M., Reliability of Computer Systems and Networks: Fault Tolerance, Analysis, and Design, Wiley, 2002.

[5] Dudin, A. N., Sun, B.' A multiserver MAP/PH/N system with controlled broadcasting by unreliable servers // Automatic Control and Computer Sciences 2009, No 5, pp 32-44.

[6] .Vishnevskii, V.M., Teoreticheskie osnovy proektirovaniya (Theoretical Foundations of Design), Moscow: Tekhnosfera, 2003

[7] Aliev, T.I. The synthesis of service discipline in systems with limits // Communications in Computer and Information Science, IET - 2016, vol. 601, pp. 151-156.

[8] Korobeynikov A.G., Fedosovsky M.E., Maltseva N.K., Baranova O.V., Zharinov I.O., Gurjanov A.V., Zharinov O.O. Use of Information Technologies in Design and Production Activities of Instrument-Making Plants//Indian Journal of Science and Technology, IET - 2016, vol. 9, No. 44, pp. 104-108.

[9] Kolomoitcev, V.S., Bogatyrev, V.A. The fault-tolerant structure of multilevel secure access to the resources of the public network // Communications in Computer and Information Science, IET - 2016, vol. 678 , pp. 302-313.

[10] Bogatyrev, V.A., Bogatyrev, A.V. Functional Reliability of a RealTime Redundant Computational Process in Cluster Architecture Systems //Automatic Control and Computer Sciences. 2015, vol. 49. No. 1, pp. 46-56. DOI 10.3103/S0146411615010022.

[11] Bogatyrev, V.A., Bogatyrev, S.V., Golubev, I.Y. Optimization and the Process of Task Distribution between Computer System Clusters // Automatic Control and Computer Sciences 2012, vol. 46, No 3, pp. 103-111.

[12] Bogatyrev, V.A. Protocols for dynamic distribution of requests through a bus with variable logic ring for reception authority transfer // Automatic Control and Computer Sciences1999, vol. 33, No 1, pp. 57-63.

[13] V.A. Bogatyrev Increasing the fault tolerance of a multi-trunk channel by means of inter-trunk packet forwarding // Automatic Control and Computer Sciences 1999, vol. 33, No 2, pp. 70-76.

[14] Bogatyrev V.A. On interconnection control in redundancy of local network buses with limited availability // Engineering Simulation 1999, vol. 16, No 4, pp. 463-469

[15] Bogatyrev, V.A. Exchange of Duplicated Computing Complexes in Fault tolerant Systems // Automatic Control and Computer Sciences 2011, Vol. 45, No 5, pp. 268-276.

[16] Bogatyrev, V.A., Parshutina, S.A. Redundant Distribution of Requests Through the Network by Transferring Them Over Multiple Paths //Communications in Computer and Information Science, IET 2016, vol. 601, pp. 199-207.

[17] Bogatyrev, V.A., Parshutina, S.A. Efficiency of Redundant Multipath Transmission of Requests Through the Network to Destination Servers // Communications in Computer and Information Science, IET - 2016, vol. 678, pp. 290-301.

[18] .Bogatyrev, V.A., Parshutina, S.A., Poptcova, N.A., Bogatyrev, A.V. Efficiency of Redundant Service with Destruction of Expired and Irrelevant Request Copies in Real-Time Clusters // Communications in Computer and Information Science, IET - 2016, vol. 678, pp. 337 348 .

[19] Bogatyrev, V.A. An interval signal method of dynamic interrupt handling with load balancing // Automatic Control and Computer Sciences 2000, vol. 34, No 6, pp. 51-57.

[20] Bogatyrev, V.A. Fault Tolerance of Clusters Configurations with Direct Connection of Storage Devices // Automatic Control and Computer Sciences, 2011, vol. 45, No 6, pp. 330-337.

[21] .Arustamov, S.A., Bogatyrev, V.A., Polyakov, V.I. Back Up Data Transmission in Real-Time Duplicated Computer Systems // Advances in Intelligent Systems and Computing, IET - 2016, vol. 451, pp. 103-109.

[22] Bogatyrev V.A., Slastikhin I.A. Redundancy of transmissions over the aggregated channels divided into groups. Scientific and Technical Journal of Information Technologies, Mechanics and Optics 2016 , vol. 16, No 6, pp. 1137-1140. doi: 10.17586/2226-1494-2016-16-6$1137-1140$ 